\title{
Passenger Satisfaction and Mental Adaptation under Adverse Conditions: Case Study in Manila
}

\author{
Andra Charis Mijares, Mio Suzuki, and Tetsuo Yai \\ Tokyo Institute of Technology
}

\begin{abstract}
Public transportation systems in several developing cities face congestion, air pollution, and safety problems, yet many passengers use them regularly. This study examines the structure of passenger satisfaction and the role of mental adaptation under such conditions. Metro Manila MRT-3 was analyzed as a case study.

The actual and perceived conditions at the MRT-3 were assessed using surveys. Results of the waiting time and $P M_{2.5}$ monitoring surveys revealed that passengers queue for 30 minutes, on average, while being exposed to unsafe levels of $P M_{2.5}$. The questionnaire survey results show some discrepancies between actual and perceived values, suggesting a perception gap.

Passenger satisfaction in MRT-3 was then modeled using ordered logit, with actual and perceived conditions (waiting time, in-vehicle time, fare levels, risk perception, and air quality perception) as significant explanatory variables. Mental adaptation was found to moderate passenger satisfaction, which may explain why some passengers are satisfied despite MRT-3's shortcomings.
\end{abstract}

Keywords: Commute satisfaction, waiting time, mental adaptation, $P M_{2.5}$ exposure, risk perception, air quality perception

\section{Introduction}

Efficient mass public transportation systems in developing cities are essential to address increasing mobility needs. However, their level of service is typically characterized by chronic congestion, unreliability and safety problems (National Research Council 1996), which result from various factors such as insufficient and outdated infrastructure, inadequate planning, and lack of safety measures. Moreover, rapid urbanization and 
inadequate environmental measures in many developing cities have led to the increase of air pollutant emissions and deteriorating urban air quality (Kim Oanh et al. 2006). In line with this, air pollution in public transportation systems is an increasing cause of concern and air pollutant exposure depends on the travel mode used (e.g., Chan et al. 2002, Niewenhuijsen et al. 2007).

These conditions likely could lead to lost productivity and opportunity costs, stress and anxiety, health impacts, and accidents. However, in spite of these negative physiological and psychological effects, many commuters still endure this situation daily. One possible explanation is lack of choice, so physical adaptation (e.g., changing departure time or mode) would no longer be feasible. Another possible explanation is a psychological phenomenon called mental adaptation, which refers to the desensitization to a negative stressor with repeated exposure over time. It is similar to hedonic adaptation in psychology, which is defined as the psychological process by which people become accustomed to a positive or negative stimulus, such that the emotional effects of that stimulus are attenuated over time (Frederick and Loewenstein 1999). In this context, it would imply that commuters may have become accustomed to commuting in such conditions due to repeated exposure and have changed their way of thinking about their commute, which helps reduce its negative psychological effects. In the same vein, some researchers have found that travel mode choice also depends on psychological factors rather than just the objective service level of the transportation system (Fujii and Kitamura 2003).

Moreover, the traditional way of evaluating passenger satisfaction may not suffice for such systems, as it focuses on conventional level-of-service attributes such as comfort, convenience, and accessibility that are more relevant to the developed world, where transportation systems usually have better service quality and commuters are used to higher standards. In the case of severe commuting conditions, it may be more appropriate to employ a vaster approach that considers the distinct problems faced.

Several studies have focused on commute satisfaction in developing countries, which typically have inadequate infrastructure and are more polluted than developed ones. Rahaman and Rahaman (2009) found that overall service satisfaction in a railway section in Bangladesh depends on factors including waiting time, crowding, and security. Ngatia et al. (2010) noted that commute satisfaction in Nairobi is significantly influenced by travel cost, service quality, and safety. Tangphansankun et al. (2010) found that fare, comfort, convenience, safety, and security are the main explanatory variables for commute satisfaction on Bangkok's paratransit modes.

Taking these into consideration, this paper investigates the structure of passenger satisfaction considering actual and perceived conditions. Specifically, it includes pollution exposure, waiting time, risk perception, fare levels, in-vehicle travel time, and adaptation as predictors of passenger satisfaction using Metro Manila MRT-3 as a case study. Various data collection methods are used to establish the extent of the congestion, air pollution, and safety problems, then a passenger satisfaction model that tests the significance of the above-mentioned predictors is developed. The perceptionbased approach in this paper is deemed to be more appropriate given the situation 
wherein commuters have physically adapted to their situation and that their only option left is to change their way of thinking about their commute (Mijares et al. 2016).

\section{Case Study: Metro Manila MRT-3}

Metro Manila is the chief metropolitan area in the Philippines. It has a daytime population of 14.5 million in a $638.6 \mathrm{~km}^{2}\left(246.6 \mathrm{mi}^{2}\right)$ area, yet its rail network is underdeveloped. Among its rail lines, Metro Rail Transit Line 3 (blue line in Figure 1), or MRT-3, has the highest ridership and is generally considered as the most critical. The 16.7-km (10.4-mi) line runs along EDSA, which is Metro Manila's main thoroughfare that connects major central business districts and other landmarks. However, its level of service has been deteriorating since 2005 when its ridership exceeded its design capacity. The only other public transportation options along EDSA are air-conditioned and ordinary buses, which are slow due to frequent stopping and heavy traffic congestion and are more expensive, especially for longer trips. Jeepneys, which are the most heavily-used public transportation mode in Metro Manila, are not permitted to ply along EDSA, but they are essentially smaller versions of ordinary buses as they are also diesel-run and open-air.

FIGURE 1.

Existing rail network in

Metro Manila

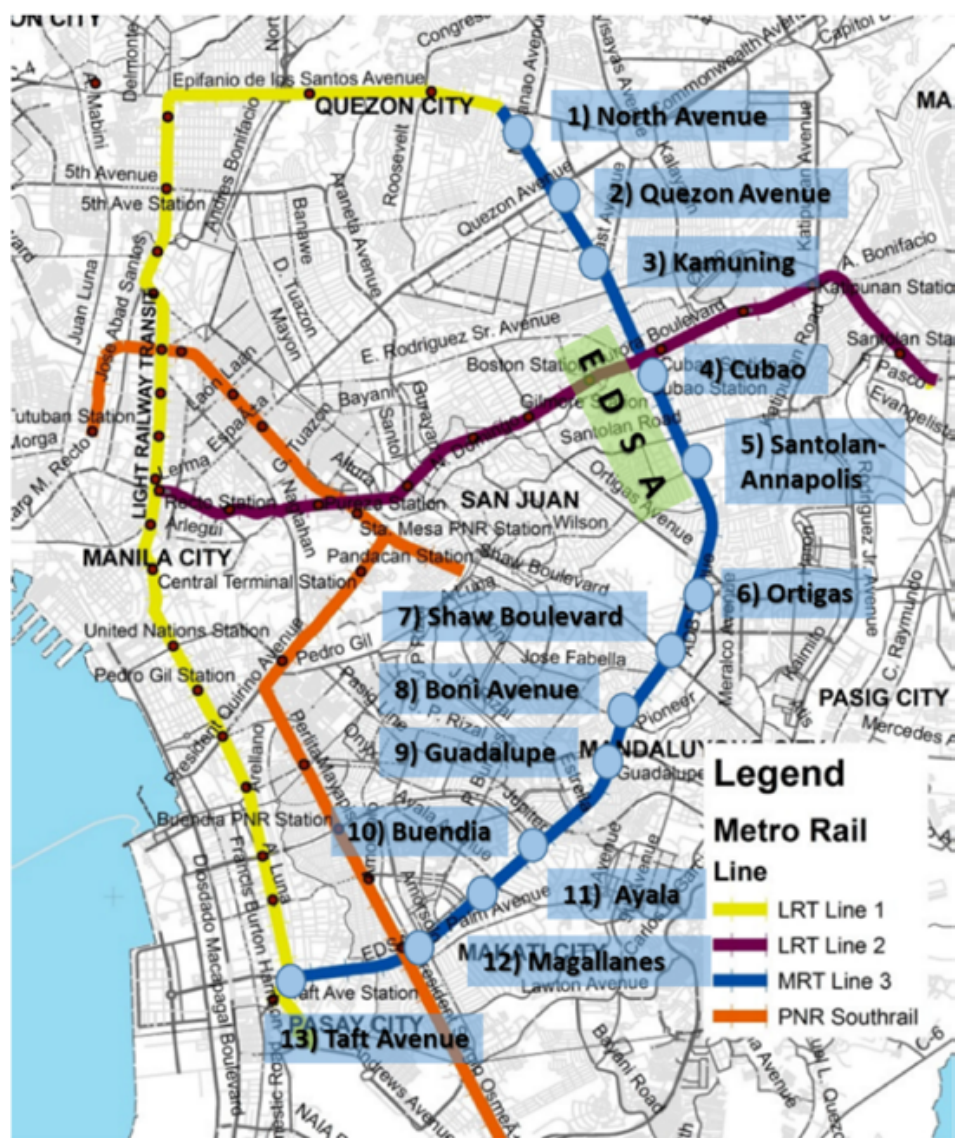


This problem is multi-faceted and encompasses financial, political, and institutional barriers, but it is mostly attributed to insufficient capacity relative to passenger demand. Significant changes in infrastructure and operations to increase its capacity have not been implemented since its full operations began in 2000. Urban rail fares also had been kept constant from 2000 to 2014 amid inflation and increases in non-rail public transportation fares, which made urban rail travel relatively cheaper, contributing to the increase of rail demand and deteriorating level of service.

As a result of the discrepancy between passenger demand and MRT-3 supply, many passengers spend a long time waiting at several stations during morning rush hours. Also, there have been several safety incidents in the past few years, with the most severe being a derailment accident in August 2014. Moreover, exposure to particulate air pollution is also a matter of concern for Metro Manila dwellers, especially regular commuters. Whereas coarse particle (PM10) concentration levels in Metro Manila are monitored daily by the government and are generally within the 24-hour guideline values, such monitoring is not yet fully implemented for fine particles $\left(\mathrm{PM}_{2.5}\right)$ even though they have worse health effects. Moreover, previous studies suggest that $\mathrm{PM}_{2.5}$ concentration levels in Metro Manila are much higher than the guideline values especially in high traffic areas (Kim Oanh et al. 2006), and at the roadside and platform of an MRT-3 station (Simpas et al. 2011). As such, commuters may be exposed to unhealthy $\mathrm{PM}_{2.5}$ levels for a prolonged period while waiting at the roadside and platform of the MRT-3.

Despite these problems, ridership is still high because the MRT-3's level of service is relatively superior to other modes in terms of affordability, travel time, safety, and accessibility, which, in a sense, leaves commuters with no choice but to continue using MRT-3 daily. Another reason is mental adaptation, which was confirmed to moderate commuting stress for some passengers when using MRT-3 (Mijares et al. 2016); however, its effect on overall passenger satisfaction has not been studied.

\section{Data Collection}

Three different types of surveys were conducted to establish the conditions at the MRT3: (1) waiting time and in-vehicle travel time surveys, (2) PM2.5 particle count monitoring survey, and (3) a questionnaire survey.

\section{Waiting Time and In-vehicle Travel Time Surveys}

An observation survey was conducted on October 1, 2014 (Wednesday) to determine the extent of passenger waiting time, which is the time spent waiting from the end of the queue into the station up to getting on the train. A surveyor was deployed as an MRT-3 passenger at every 15-minute interval from 6:45-8:00 AM at North Avenue and Cubao stations, and the time spent completing each stage of queuing was recorded. Meanwhile, an in-vehicle travel time survey was performed on 20 regular weekdays between February-March 2015 for MRT-3 as well as ordinary and air-conditioned buses for intermodal comparison. 


\section{PM2.5 Particle Count Monitoring Survey}

This survey aimed to quantify the $\mathrm{PM}_{2.5}$ exposure while waiting at the roadside and platform of MRT-3 stations and while inside the train, as well as to compare the results with those of buses along EDSA. The survey was divided into two components: intramodal and intermodal. Intra-modal comparison focused on the roadside, ticketing area and platform of five out of 13 MRT-3 stations, which were selected according to their morning peak ridership and characteristics. Intermodal comparison was conducted by measuring the $\mathrm{PM}_{2.5}$ levels while inside the train or bus.

PM2.5 levels were measured using particle count using a portable particle counter that measures the number of particles detected through light scattering and produces instantaneous results. Particle count is an equally important indicator of air quality as mass concentration, especially in investigating associations between air pollution and adverse health outcomes (Ruuskanen et al. 2001). It is even suggested to be more closely correlated to adverse health effects than mass concentration (Wichmann et al. 2000). That said, the typically-used mass concentration would have also been appropriate as PM 2.5 count includes the more harmful ultra-fine particles (>1 micrometer), but it is costlier and more time-consuming to measure.

PM2.5 particle count was measured at every one-minute interval during the morning peak period on 20 regular weekdays from February to March 2015 using the portable particle counter. Measurements were not done simultaneously for different MRT-3 stations and travel modes due to equipment availability issues. Measurements were done at each travel mode or station at least four times throughout the survey period.

\section{Questionnaire Survey}

A questionnaire survey was used to assess the commute characteristics of MRT-3 passengers and their perception on air quality, risk and adaptation. It mainly targeted commuters who use the MRT-3 during the morning peak period on a daily basis to travel from their home to the workplace. Data collection was conducted in September 2014 in cooperation with the University of the Philippines National Center for Transportation Studies (UP NCTS) using online and on-site interviews. The profile of the sample data (age and gender) was found to adequately represent that of the general population of Metro Manila. Data screening was also performed to eliminate unengaged respondents and outliers, reducing the sample size from 225 to 211 .

Among the 211 respondents, 119 (56.4\%) were females, 84 (39.8\%) had a monthly income of PhP20,000, and 130 (61.6\%) were below age 30. In total, 55 (26.1\%) respondents had been using the MRT-3 for their everyday morning commute for more than five years, and 48 (22.7\%) had been using it for less than two years. 


\section{Actual and Perceived Conditions at MRT-3}

This section establishes the extent of the congestion problem in Metro Manila MRT-3 in terms of waiting time, in-vehicle time, fare, $\mathrm{PM}_{2.5}$ exposure, and passengers' commute characteristics and perception about risk, air quality, and adaptation, which are hypothesized to be the explanatory variables that influence passengers' satisfaction with their MRT-3 commute. Comparisons with buses that run parallel to the MRT-3 alignment were also made.

\section{Passenger Waiting Time}

Passenger waiting time refers to the time spent from arriving at the end of the queue at the station until getting on the train and is the sum of station access time and platform waiting time. Platform waiting time at the MRT-3 has been studied and shown to be disproportionately high in the middle stations (Mijares et al. 2013, 2014). Station access time is also a critical part of total waiting time as ocular surveys have shown that queues into the station frequently spill out onto the roadside. Furthermore, MRT-3 passengers are not provided real-time information by the operator about their estimated waiting time.

The results of the waiting time survey in October 2014 indicated that passengers experience long and variable waiting times at certain stations as a result of the O-D patterns and operations policies. These include the "stop entry" policy, which limits the number of passengers on the platform to 500 at a time, and the "skip train" policy, which deploys empty trains to the third and fourth stations to provide capacity to boarding passengers (refer to Mijares et al. 2015 for more details). It was found that morning peak period passengers at North Avenue Station (northern terminal) wait for an average of 35.6 minutes, with majority of the time spent queuing at the roadside and at the stairways. Figure 2 shows the estimated cumulative arrival and departure curves at North Avenue Station, which indicates that the queue length had reached up to around 3,500 passengers during the 90 -minute interval. Meanwhile, passengers at Cubao Station (fourth station southbound) spent an average of 50.2 minutes queuing both at the roadside and platform. In general, the first five stations in the southbound (peak) direction, experience such severe waiting conditions. 
FIGURE 2.

Estimated cumulative roadside arrival and platform

departure curves at North

Avenue Station

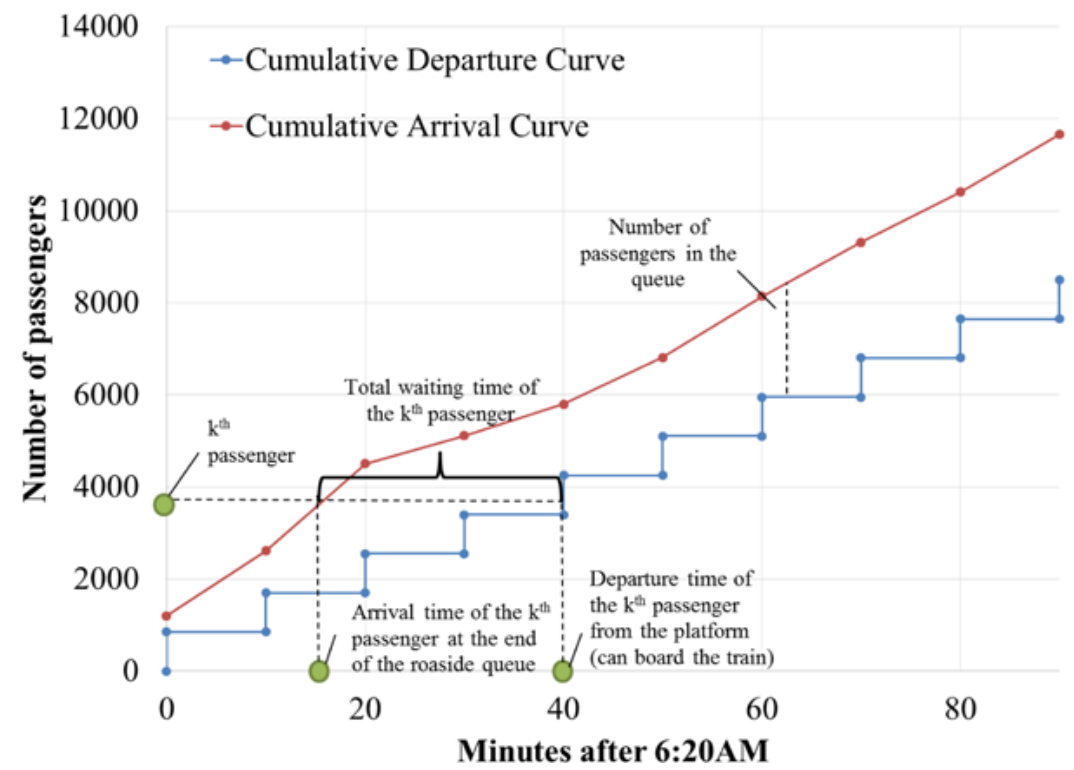

The results of the questionnaire survey are also consistent with these findings. Respondents were found to spend an average total waiting time of 30.0 minutes (Table 1). Waiting time variability at the MRT- 3 was also an issue largely as a result of the operations policies, with $33.6 \%$ of respondents stating that their waiting time varied by $10-20$ minutes on average and $48.3 \%$ reporting that it varied by more than 20 minutes on average.

TABLE 1.

MRT-3 Commute Characteristics of Respondents

\begin{tabular}{|c|r|r|r|r|}
\hline \multicolumn{1}{|c|}{ Variable } & \multicolumn{1}{c|}{ Min. } & \multicolumn{1}{c|}{ Max. } & \multicolumn{1}{c|}{ Mean } & Std. Dev. \\
\hline Total waiting time at MRT-3 & $5 \mathrm{~min}$ & $60 \mathrm{~min}$ & $30.0 \mathrm{~min}$ & $15.2 \mathrm{~min}$ \\
\hline Feeder time (access and egress) & $3 \mathrm{~min}$ & $180 \mathrm{~min}$ & $41.4 \mathrm{~min}$ & $26.7 \mathrm{~min}$ \\
\hline In-vehicle travel time at MRT-3 & $4.5 \mathrm{~min}$ & $40 \mathrm{~min}$ & $28.1 \mathrm{~min}$ & $10.9 \mathrm{~min}$ \\
\hline Total trip time & $30 \mathrm{~min}$ & $240 \mathrm{~min}$ & $118.4 \mathrm{~min}$ & $41.3 \mathrm{~min}$ \\
\hline MRT-3 fare & PhP10.0 & PhP15.0 & PhP12.7 & PhP1.5 \\
\hline Feeder fare (access and egress) & PhP0.0 & PhP90.0 & PhP33.8 & PhP15.0 \\
\hline Total fare & PhP11.0 & PhP102.0 & PhP45.5 & PhP13.5 \\
\hline
\end{tabular}

\section{In-Vehicle Travel Time and Feeder Travel Time}

The results of the in-vehicle travel time survey show that MRT-3 had an average speed of $23.5 \mathrm{kph}$ considering running and dwell times, but dropped to around $16.1 \mathrm{kph}$ when waiting time was included. In comparison, ordinary and air-conditioned buses along the same route had average speeds of $10.9 \mathrm{kph}$ and $11.2 \mathrm{kph}$, respectively, due to road congestion and frequent stops.

However, MRT-3 commuters typically have to use feeder modes as well, which are commonly road-based. The questionnaire survey revealed that while in-vehicle travel time using the MRT-3 is at an average of 28.1 minutes, the average feeder access time 
is 41.4 minutes (Table 1), with an average of 2.7 transfers, bringing the average total morning commute time to almost two hours (Table 1). Overall, this indicates that MRT-3 commuters spend a long time commuting and that a substantial part of it is spent on waiting at the MRT- 3 and feeder modes.

\section{Fare Levels}

When the survey was conducted in 2014, fare levels in MRT-3 ranged from PhP10 for the first three stations ( 4 km) and PhP15 for an end-to-end trip ( 17 km). In contrast, road-based transportation modes had higher fares, with bus fares almost double the MRT-3 fares for longer trips. The fare structure is distance-based but not integrated (i.e., need to pay base fare for every transfer), making the whole trip cost higher especially for people who live or work far from the MRT-3 line.

\section{Air Quality}

The analysis of air quality in MRT-3 is limited to PM2.5 particle count, which may pose health risks, and passengers' perception on air quality.

PM2.5 Particle Count Measurement. Intra-modal comparison was conducted at roadside, stairways, ticketing areas, and platform of five out of 13 MRT-3 stations (Figure 1). The average particle count per minute $(\mathrm{pcm})$ are as follows: (1) North Avenue $-78.9 \mathrm{pcm}$ ( $\mathrm{sd}=32.5 \mathrm{pcm}$ ); (2) Quezon Avenue $-82.1 \mathrm{pcm}(\mathrm{sd}=34.4 \mathrm{pcm}$ ); (3) Cubao $-79.7 \mathrm{pcm}$ (sd $=27.2 \mathrm{pcm}$ ), (4) Ayala $-70.5 \mathrm{pcm}(\mathrm{sd}=22.9 \mathrm{pcm})$; and (5) Taft Avenue $-106.8 \mathrm{pcm}$ (sd $=45.8 \mathrm{pcm}$ ). Taft Avenue, which is located near provincial bus terminals, has significantly higher pcm than the other stations.

Intermodal comparison between MRT-3, ordinary bus and air-conditioned bus entailed measurements of $\mathrm{PM}_{2.5}$ particle counts inside the vehicles and exposure time (equivalent to running time and dwell time). Ordinary buses had the highest $\mathrm{PM}_{2.5}$ levels ( $m e a n=108.7 \mathrm{pcm}, \mathrm{sd}=71.9 \mathrm{pcm}$ ), and the longest in-vehicle travel time for a $16.7-\mathrm{km}$ trip (mean running time $=77.1 \mathrm{~min}$, mean dwell time $=15.1 \mathrm{~min}$ ), making it the worst mode among the three in terms of $\mathrm{PM}_{2.5}$ exposure. Air-conditioned buses ranked second, with $\mathrm{PM}_{2.5}$ levels (mean $=56.3 \mathrm{pcm}, \mathrm{sd}=40.4 \mathrm{pcm}$ ) that are considerably lower than that of ordinary buses but slightly higher and more variable than that of MRT-3 (mean=53.7 $\mathrm{pcm}, \mathrm{sd}=23.9 \mathrm{pcm}$ ). In-vehicle travel time of air-conditioned buses was almost similar to that of ordinary buses (mean running time $=63.0 \mathrm{~min}$, mean dwell time $=26.3 \mathrm{~min}$ ), thus exposure time was also around 90 minutes for a one-way trip.

When additional exposure when waiting is considered, exposure time is equivalent to the sum of waiting, running and dwell times. Even in this case, ordinary buses still had the highest $\mathrm{PM}_{2.5}$ levels and longest exposure time among the three modes, as seen in Figure 3. However, the ranking between air-conditioned bus and MRT-3 switched because the $\mathrm{PM}_{2.5}$ levels while waiting at the roadside, ticketing area, and platforms drove up the overall exposure levels for MRT-3 (mean $=65.9 \mathrm{pcm}, \mathrm{sd}=28.6 \mathrm{pcm}$ ). In contrast, $\mathrm{PM}_{2.5}$ exposure for bus passengers mostly occurred while inside the vehicles given that buses ran frequently along EDSA so waiting time was significantly lower. 
FIGURE 3.

Intermodal comparison of $\mathrm{PM}_{2.5}$ exposure (travel time and particle count)
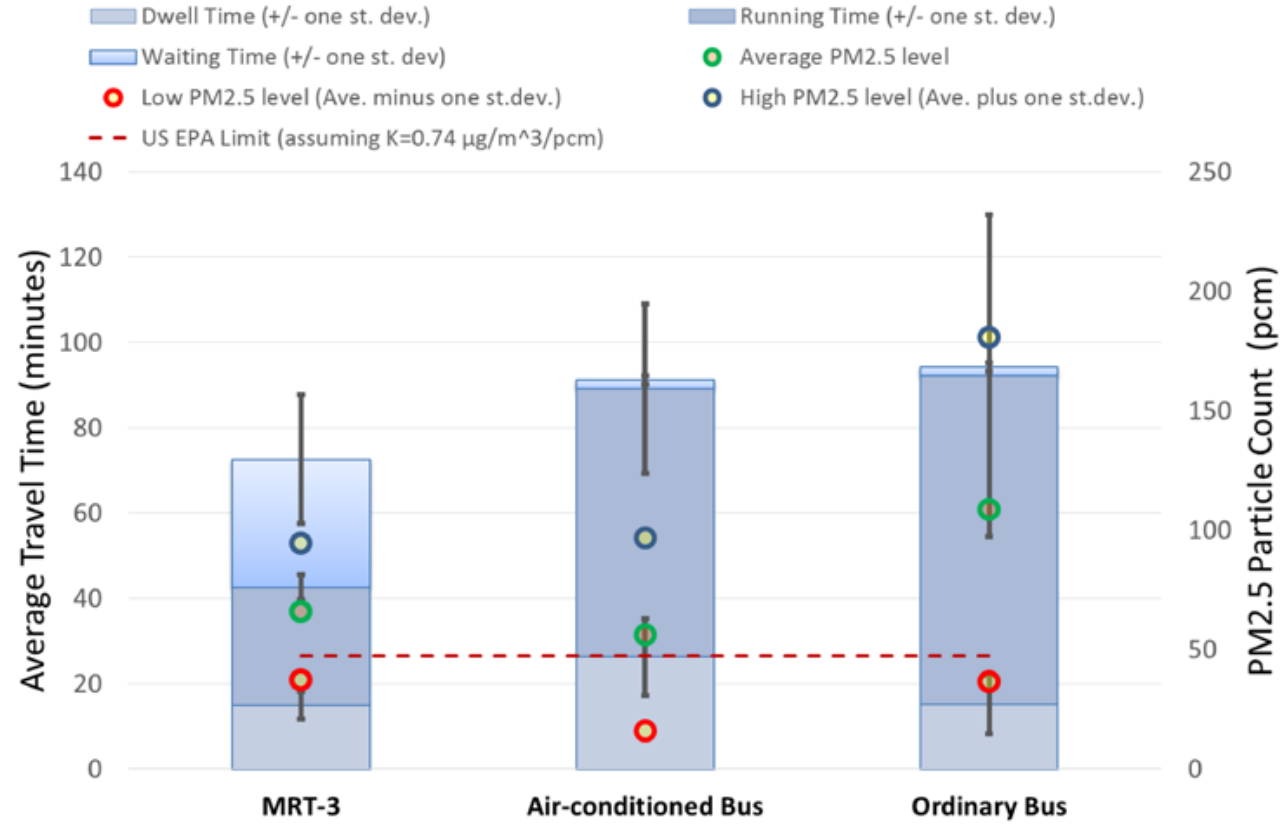

To allow a comparison with the US EPA limit in Figure 3, a conversion factor that converts particle count to mass concentration was calculated based on fine particle characteristics in Metro Manila (Simpas et al. 2011) and particle density. Using this conversion factor, the USA EPA limit of $35 \mu \mathrm{g} / \mathrm{m}^{3}$ would be equivalent to $47 \mathrm{pcm}$.

A comparison with $\mathrm{PM}_{2.5}$ measurements in some sites was also made for reference. At a rural town outside Metro Manila called Gabaldon, Nueva Ecija, the average $\mathrm{PM}_{2.5}$ concentration was $11 \mu \mathrm{g} / \mathrm{m}^{3}$ ( 14.8 pcm) (Simpas et al. 2011), which is way below the US EPA limit and the EDSA values. Measurements were also made by the survey team using the same equipment at Laurel Avenue in Metro Manila, which is along a jeepney route and has moderate to heavy vehicle traffic. The average value is $103.1 \mathrm{pcm}$, which is similar to the level in ordinary buses and roadside of MRT-3. Jeepneys are popular MRT-3 feeder modes, so the findings suggest that MRT-3 commuters are exposed to unhealthy $\mathrm{PM}_{2.5}$ levels in other aspects of their commute.

Air Quality Perception. Perceived air quality pertains to passengers' rating on the air quality at the MRT-3, and represents their awareness and concern about their exposure to air pollution during their commute. PM PM.5 $_{2}$ exposure is substantial for MRT-3 commuters as established in the $\mathrm{PM}_{2.5}$ monitoring survey.

PM 2.5 particle count and waiting time were correlated against air quality perception, which is measured by the reverse of the statement, "I am exposed to air pollution while waiting to ride the MRT-3 at the roadside, ticketing area and platform" using a 7-point Likert scale (mean=5.3; sd=1.8); however, no significant relationships were found. The lack of statistically significant correlations implies that air quality perception is linked to other individual differences rather than exposure-related measurable data. This finding is consistent with previous studies which show that the visual and olfactory characteristics of air have a significant impact on perceived air quality, so the absence of 
black exhaust fumes (like PM2.5, which is invisible to the naked eye) may lead to a better rating of air quality (Saksena 2011).

\section{Safety and Risk Perception}

Risk perception is characterized as the intuitive judgment of individuals and groups of risks in the context of limited and uncertain information (Slovic et al. 1985). It is difficult for non-experts to correctly perceive objective safety, so there may be a gap between the two.

Respondents were asked to rate risk perception in the questionnaire survey using the reverse of the statement, "I feel that MRT-3 is a safe transport mode" using a 7-point Likert scale (mean=4.2, $s d=1.5$; not reversed). The results imply that respondents have moderate ratings of perceived risk.

\section{Adaptation}

There are two types of adaptation in commuting: (1) physical (or behavioral) adaptation, which means changing their behavior or the situation itself; and (2) mental adaptation, which refers to changing their way of thinking about it (Punpuing and Ross 2001).

The questionnaire survey results confirmed that passengers had already physically adapted to the situation by changing their travel behavior in one or more ways-90\% had switched to an earlier departure time, $19 \%$ had changed their boarding station to a less crowded one, $19 \%$ had moved to another residence, and $5 \%$ had moved to another workplace.

As MRT-3 commuters had already exhausted physical adaptation options, mental adaptation may set in as a coping mechanism in dealing with this unsatisfactory situation daily. Mental adaptation was measured using the statement, "I have completely adapted to commuting in this situation" (mean=3.9, $\mathrm{sd}=1.7$ ) and "I have become used to this everyday situation" (mean=4.0; $\mathrm{sd}=1.7$ ) using a 7-point Likert scale. Both statements were found to be internally consistent, but the first statement was used to represent mental adaptation for the purpose of analysis. Results indicate that mental adaptation had not set in for many respondents. Contrary to intuition, there was no correlation between adaptation and the length of experience with using MRT-3 daily, implying that adaptation may be due to individual characteristics.

\section{Passenger Satisfaction Model}

This section investigates the structure of passenger satisfaction in MRT-3 considering the actual and perceived conditions discussed in the previous section. Fare, in-vehicle travel time, waiting time and its variability, perception on risk and air quality, and mental adaptation are hypothesized to influence passengers' satisfaction with their MRT-3 commute (Table 2). 
TABLE 2. Proposed Explanatory Variables and Expected Results

\begin{tabular}{|c|c|c|c|c|}
\hline \multirow[b]{2}{*}{ Explanatory Variable } & \multirow{2}{*}{$\begin{array}{l}\text { Variable } \\
\text { Name }\end{array}$} & \multirow{2}{*}{$\begin{array}{l}\text { Variable } \\
\text { Type }\end{array}$} & \multicolumn{2}{|c|}{ Expected Results } \\
\hline & & & General & $\begin{array}{l}\text { Differences between } \\
\text { Income Groups }\end{array}$ \\
\hline $\begin{array}{l}\text { Total fare in Philippine pesos (MRT-3 } \\
\text { and feeder modes) }\end{array}$ & $C$ & Continuous & $\begin{array}{l}\text { Lower fare } \rightarrow \text { Higher } \\
\text { satisfaction rating }\end{array}$ & $\begin{array}{l}\text { Low income } \rightarrow \text { higher } \\
\text { effect }\end{array}$ \\
\hline $\begin{array}{l}\text { Total in-vehicle travel time in } \\
\text { minutes (MRT-3 and feeder modes) }\end{array}$ & $T$ & Continuous & $\begin{array}{l}\text { Lower in-vehicle travel time } \rightarrow \\
\text { Higher satisfaction rating }\end{array}$ & $\begin{array}{l}\text { Medium/High income } \\
\rightarrow \text { higher effect }\end{array}$ \\
\hline $\begin{array}{l}\text { Average waiting time at the MRT-3 } \\
\text { in minutes }\end{array}$ & W & Continuous & $\begin{array}{l}\text { Lower waiting time } \rightarrow \text { Higher } \\
\text { satisfaction rating }\end{array}$ & $\begin{array}{l}\text { Medium/High income } \\
\rightarrow \text { higher effect }\end{array}$ \\
\hline $\begin{array}{l}\text { Waiting time variability ( } 1 \text {-always } \\
\text { the same; } 5 \text { - more than } 30 \text { minutes) }\end{array}$ & V & $\begin{array}{l}\text { Ordinal } \\
(1-5 \text { scale })\end{array}$ & $\begin{array}{l}\text { Lower variability } \rightarrow \text { Higher } \\
\text { satisfaction rating }\end{array}$ & $\begin{array}{l}\text { Medium/High income } \\
\rightarrow \text { higher effect }\end{array}$ \\
\hline Air quality perception & Q & $\begin{array}{l}\text { Ordinal } \\
\text { (1-7 scale) }\end{array}$ & $\begin{array}{l}\text { Higher air quality perception } \\
\rightarrow \text { Higher satisfaction rating }\end{array}$ & $\begin{array}{l}\text { Medium/High income } \\
\rightarrow \text { higher effect }\end{array}$ \\
\hline Risk perception & $R$ & $\begin{array}{l}\text { Ordinal } \\
\text { (1-7 scale) }\end{array}$ & $\begin{array}{l}\text { Lower risk perception } \rightarrow \\
\text { Higher satisfaction rating }\end{array}$ & $\begin{array}{l}\text { Medium/High income } \\
\rightarrow \text { higher effect }\end{array}$ \\
\hline Mental Adaptation Level & A & $\begin{array}{l}\text { Ordinal } \\
\text { (1-7 scale) }\end{array}$ & $\begin{array}{l}\text { Higher mental adaptation level } \\
\rightarrow \text { Higher satisfaction rating }\end{array}$ & No difference \\
\hline
\end{tabular}

The ordered logit model was chosen to represent passenger satisfaction because it is more appropriate for ordered data and has been used in general satisfaction studies (e.g., Theodossiou, 1998). Passenger satisfaction was measured by the statement, "I am satisfied with the service provided by MRT-3" using a 7-point Likert scale. This was reduced to a 4-point scale to reduce the skewness of the data, where 1-strongly dissatisfied, 2-dissatisfied, 3-neutral, and 4-satisfied.

The passenger satisfaction model has the proposed form:

$$
y_{i}^{*}=\beta_{c} C_{i}+\beta_{t} T_{i}+\beta_{w} W_{i}+\beta_{v} V_{i}+\beta_{Q} Q_{i}+\beta_{r} R_{i}+\beta_{a} A_{i}+\varepsilon_{i}
$$

For all $i=1, \ldots, N$; in which the continuous latent utility, $y_{i}^{*}$, (passenger satisfaction) is observed in discrete form through a censoring mechanism. The expected results are shown in Table 2.

Data from the questionnaire survey was used to estimate the model. Income segmentation was done: low income (monthly salary $\leq$ PhP20,000) and medium- to high-income (monthly salary $>$ PhP20,000). Low-income respondents had higher satisfaction levels than their counterparts, probably because MRT-3 is relatively affordable. More adapted commuters were also more satisfied, confirming that mental adaptation plays a role in improving satisfaction.

The model estimates for the different models are provided in Table 3. All relevant tests (e.g., chi-square, test of parallel lines) were acceptable for all models. The results in Table 3 are similar to the expected results in Table 2, except for waiting time variability which was insignificant. Skip train service might also have a role in explaining its insignificance, as passengers may have become habituated to the day-to-day variability of waiting time 
resulting from this policy (Mijares et al. 2014). Total fare was also valued more by lowincome passengers as higher travel costs would mean less monetary resources for other needs.

TABLE 3. Parameter Estimates for Passenger Satisfaction Model

\begin{tabular}{|c|c|c|c|c|c|c|c|c|c|}
\hline \multirow{3}{*}{ Model } & \multirow{3}{*}{$\begin{array}{l}-2 \text { Log- } \\
\text { Likelihood } \\
\text { (Final) }\end{array}$} & \multirow{3}{*}{$\begin{array}{c}\text { Model } \\
\text { Fit/ } \\
\text { Test of } \\
\text { Parallel } \\
\text { Lines }\end{array}$} & \multicolumn{7}{|c|}{ Parameter Estimates, (Level of Significance) } \\
\hline & & & \multicolumn{7}{|c|}{ Predictor Variables } \\
\hline & & & $\begin{array}{l}\text { Total } \\
\text { Fare }\end{array}$ & $\begin{array}{c}\text { Total } \\
\text { In-Vehicle } \\
\text { Travel Time }\end{array}$ & $\begin{array}{l}\text { Waiting } \\
\text { Time }\end{array}$ & $\begin{array}{l}\text { Waiting } \\
\text { Time } \\
\text { Variability }\end{array}$ & $\begin{array}{l}\text { Air Quality } \\
\text { Perception }\end{array}$ & $\begin{array}{c}\text { Risk } \\
\text { Perception }\end{array}$ & $\begin{array}{c}\text { Mental } \\
\text { Adaptation }\end{array}$ \\
\hline Full model $(\mathrm{N}=211)$ & 401.3 & OK & $\begin{array}{c}-0.062 \\
(1 \%)\end{array}$ & $-0.018(1 \%)$ & $\begin{array}{c}-0.072 \\
(1 \%)\end{array}$ & 0.19 (NS) & $0.23(1 \%)$ & $-0.37(1 \%)$ & $0.40(1 \%)$ \\
\hline $\begin{array}{l}\text { Low-income group } \\
(\mathrm{N}=84)\end{array}$ & 150.2 & OK & $\begin{array}{l}-0.13 \\
(1 \%) \\
\end{array}$ & $-0.013(5 \%)$ & $\begin{array}{c}-0.070 \\
(5 \%)\end{array}$ & 0.23 (NS) & $0.33(5 \%)$ & $-0.31(10 \%)$ & $0.20(5 \%)$ \\
\hline $\begin{array}{l}\text { Medium-/ high- } \\
\text { income group }(\mathrm{N}=127)\end{array}$ & 233.7 & OK & $\begin{array}{c}-0.032 \\
(5 \%)\end{array}$ & $-0.019(1 \%)$ & $\begin{array}{c}-0.079 \\
(5 \%)\end{array}$ & 0.26 (NS) & $0.21(5 \%)$ & $-0.39(1 \%)$ & $0.47(1 \%)$ \\
\hline
\end{tabular}

NS - non-significance

The values of in-vehicle time and waiting time were calculated by dividing the coefficients of in-vehicle time and waiting time by that of the total fare. Table 4 shows that waiting time was valued around four times larger than in-vehicle travel time for all groups, implying a strong aversion to waiting. This estimate is slightly higher than those in previous studies (e.g., Mohring et al. 1987, Mishalani et al. 2006) wherein waiting time was valued 1.5-3 times higher than in-vehicle travel time, probably because the waiting time at the MRT-3 is typically longer and the waiting environment is generally more unfavorable. As expected, higher income passengers valued in-vehicle time and waiting time several times more than their low-income counterparts.

TABLE 4.

Values of In-vehicle and Waiting Time

\begin{tabular}{|l|c|c|}
\hline \multirow{2}{*}{ Model } & \multicolumn{2}{c|}{$\begin{array}{c}\text { "I am satisfied with the service } \\
\text { provided by MRT-3" }\end{array}$} \\
\cline { 2 - 3 } & $\begin{array}{c}\text { Value of in-vehicle } \\
\text { travel time (PhP/min) }\end{array}$ & $\begin{array}{c}\text { Value of waiting } \\
\text { time (PhP/min) }\end{array}$ \\
\hline Full model (N=211) & $0.29(1 \%)$ & $1.16(1 \%)$ \\
\hline Low-income group only (N=84) & $0.10(5 \%)$ & $0.59(5 \%)$ \\
\hline Medium-/high-income group only $(\mathrm{N}=127)$ & $0.59(1 \%)$ & $2.18(1 \%)$ \\
\hline
\end{tabular}

PhP - Philippine pesos ( 1 USD $\approx 45$ PHP)

The passenger satisfaction model was also used to estimate the changes in satisfaction levels as a result of a change in the level of the attributes, for example, due to a countermeasure. These are computed using the predicted probability equation for ordered logit model:

$$
P\left(Y_{i}>j\right)=\frac{\exp \left(X_{i} \beta-\kappa_{j}\right)}{1+\left[\exp \left(X_{i} \beta-\kappa_{j}\right)\right]}, j=1,2,3
$$

Where $\kappa_{j}$ refers to the threshold values for each category, and $j$ is the category of the passenger satisfaction level. 
A cumulative probability threshold of $55 \%$ was used to classify respondents into "strongly dissatisfied," "dissatisfied," "neutral," and "satisfied." Figure 4 shows the results of the sensitivity analysis, which illustrates the effects of an attribute change to passenger satisfaction levels compared to the baseline levels.

FIGURE 4.

Sensitivity analysis of passenger satisfaction levels

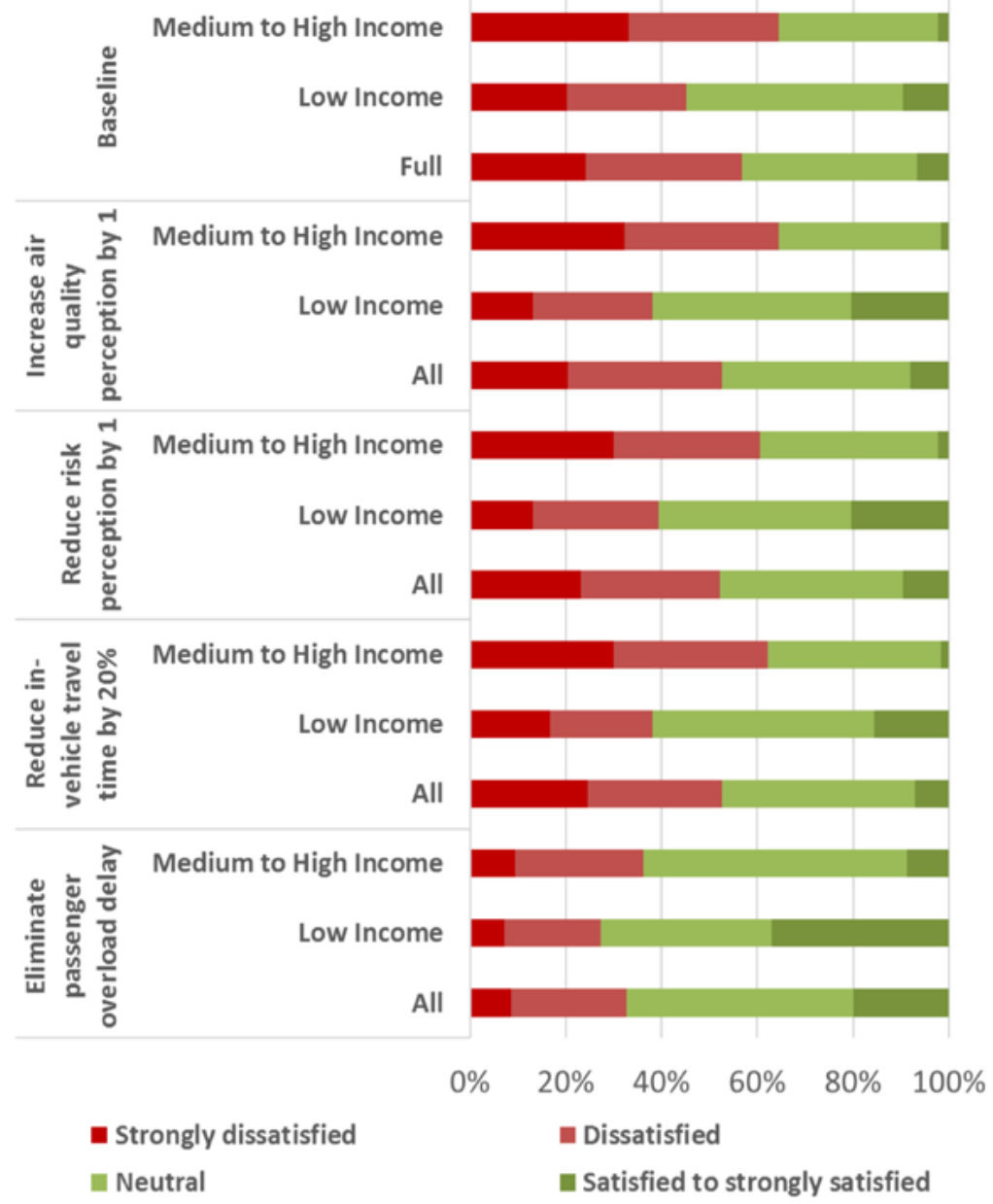

A comparison between income groups shows that low-income people generally have higher satisfaction levels than higher-income ones given that they have lower values of time.

It was found that improving perception-related variables such as air quality and risk perception would only have a minimal effect on satisfaction levels. Moreover, reducing in-vehicle travel time by $20 \%$ also yields to small changes in satisfaction levels. However, reducing waiting time by eliminating passenger overload delay (i.e., delay due to insufficient capacity) would yield the highest improvement in satisfaction, almost doubling the satisfaction levels for all groups. This implies that countermeasures that increase capacity and subsequently reduce waiting time and $\mathrm{PM}_{2.5}$ exposure should be prioritized over those that improve other variables. Some ideas for increasing capacity include adding train cars, reducing headway, and doubling the number of rail tracks. Further study needs to be done to evaluate the detailed impacts of such countermeasures. 


\section{Summary and Conclusions}

This paper examined the structure of passenger satisfaction and the role of mental adaptation in severe conditions, with MRT-3 in Metro Manila as a case study. First, it established the actual and perceived conditions at the MRT-3 in terms of waiting time, in-vehicle travel time, fare, air quality, risk perception, and adaptation using several data collection methods. Then, it developed a passenger satisfaction model that incorporates the said variables.

Passenger waiting time was found to be long and variable at the roadside and platform largely due to excessive demand and operations policies, which exposes passengers to $\mathrm{PM}_{2.5}$ for an extended period of time. The overall PM 2.5 exposure level at the MRT-3 was found to be similar to that of an air-conditioned bus due to long exposure times while waiting at the roadside and platform.

In addition, the estimation of the passenger satisfaction model using ordered logit showed that actual conditions (fare, waiting time, in-vehicle travel time) and perceived conditions (risk and air quality) predict passenger satisfaction with MRT-3. Mental adaptation tends to increase passenger satisfaction, which can be both beneficial and detrimental. On a positive note, it helps reduce the negative effects of an unfavorable commute. However, it may lower expectations and keep commuters complacent, thus they do not demand for better services from the operator, and it may also expose them to actual physiological harm.

This study would help draft appropriate countermeasures and evaluate them by extending the results of the sensitivity analysis. The results of the preliminary analysis showed that eliminating waiting time due to passenger overload delay would double passenger satisfaction levels, which suggests that increasing the capacity of MRT-3 would be an effective countermeasure.

The results also suggest that public transportation in Metro Manila should be improved in general. Even in its poor state, MRT-3 is still preferred by many commuters because of lack of appealing options. Bus services should also be improved to provide a reasonable alternative for traveling along EDSA and other parts of Metro Manila. Improving public transportation would also discourage the modal shift to private cars. Given that vehicular traffic is the main contributing factor to fine particulate matter pollution in Metro Manila (Villarin et al. 2014), reversing the rapid motorization trend by improving public transportation could contribute in improving air quality. However, solving the air pollution problem in Metro Manila would require a wider effort on a regional scale to address natural and anthropogenic sources of pollution.

Although this study specifically focuses on Metro Manila MRT-3, the methodology and evaluation framework used may also be applicable and contextualized to other public transportation systems experiencing congestion, air pollution, and safety problems especially in developing cities. 


\section{References}

Chan, L. Y., W. L. Lau, S. C. Zou, Z. X. Cao, and S.C. Lai. 2002. "Exposure Level of Carbon Monoxide and Respirable Suspended Particulate in Public Transportation Modes while Commuting in Urban Area of Guangzhong, China." Atmospheric Environment 36: 5831-5840.

Frederick, S., and G. Loewenstein. 1999. "Hedonic Adaptation." In Kahneman, D., Diener, E., and N. Schwartz (Eds.), Well-being: The Foundations of Hedonic Psychology. Russell Sage: New York, 302-329.

Fujii, S. and R. Kitamura. 2003. "What Does a One-month Free Bus Ticket Do to Habitual Drivers? An Experimental Analysis of Habit and Attitude Change." Transportation 30 (1): 81-95.

Kim Oanh, N. T., N. Upadhyay, Y. H. Zhuang, Z. P. Hao, D. V. S. Murthy, P. Lestari, J. T. Villarin, K., Chengchua, H.X. Co, N. T. Dung, and E.S. Lindgren. 2006. "Particulate Air Pollution in Six Asian Cities: Spatial and Temporal Distributions, and Associated Sources." Atmospheric Environment 40: 3367-3380.

Mijares, A. C., M. Suzuki, and T. Yai. 2013. "Equity Analysis of Urban Rail Fare Policy and Passenger Overload Delay: An International Comparison and the Case of Metro Manila MRT-3." Journal of the Eastern Asia Society for Transportation Studies 10: 45-65.

Mijares, A. C., M. Suzuki, and T. Yai. 2014. "A Study on Platform Waiting Time and Its Variability at the Metro Manila MRT-3." Proceedings of the 19th International Conference of the Hong Kong Society for Transportation Studies, 425-432.

Mijares, A. C., M. Suzuki, T. Yai., and K. N. Vergel 2015. "Actual Conditions at the Metro Manila MRT-3 during the Morning Peak Period: Focus on Passengers' Viewpoint." Proceedings of the Eastern Asia Society for Transportation Studies 10: 1361.

Mijares, A. C., M. Suzuki, and T. Yai. 2016. "An Analysis of Metro Manila MRT-3 Passengers' Perception of Their Commuting Experience and Its Effects using Structural Equation Modeling (SEM)." Asian Transport Studies 4 (1): 1-18..

Mishalani, R. G., M. M. McCord, and J. Wirtz. "Passenger Wait Time Perceptions at Bus Stops: Empirical Results and Impact on Evaluating Real-Time Bus Arrival Information." Journal of Public Transportation 9(2): 89-106.

Mohring, H., H. J. Schroeter, and P. Wiboonchutikula. 1987. "The Values of Waiting Time, Travel Time, and a Seat on a Bus." The RAND Journal of Economics, 18(1): 40-56.

National Research Council. 1996. "Transportation Options for Megacities in the Developing World." In Meeting the Challenges of Megacities in the Developing World: A Collection of Working Papers. Washington, DC: The National Academies Press, 1-4.

Ngatia, G. J., T. Okamura, and F. Nakamura. 2010. "The Structure of Users' Satisfaction on Urban Public Transport Service in a Developing Country: The Case of Nairobi." Journal of the Eastern Asia Society for Transportation Studies 8: 1288-1300. 
Nieuwenhuijsen, M. J., J. E. Gomez-Perales, and R. N. Colvile. 2007. "Levels of Particulate Air Pollution, Its Elemental Composition, Determinants and Health Effects in Metro Systems." Atmospheric Environment 41: 7995-8006.

Punpuing, S., and H. Ross. 2001. "Commuting: The Human Side of Bangkok's Transport Problems." Cities 18(1): 43-50.

Rahaman, K. R. and M. A. Rahaman 2009. "Service Quality Attributes Affecting the Satisfaction of Railway Passengers of Selective Route in Southwestern Part of Bangladesh." Theoretical and Empirical Researches in Urban Management 3(12): 115125.

Ruuskanen, J., T. Tuch, H. Ten Brink, A. Peters, A. Khlystov, A. Mirme, G. P. A. Kos, B. Brunekreef, H. E. Wichmann, G. Buzorius, M. Vallius, W. G. Kreyling, and J. Pekkanen. 2001. "Concentrations of Ultrafine, Fine and $P M_{2.5}$ Particles in Three European Cities." Atmospheric Environment 35: 3729-3738.

Saksena, S. 2011. "Public Perceptions of Urban Air Pollution Risks." Risk, Hazards \& Crisis in Public Policy 2: 1-19.

Simpas, J. B., M. Templonuevo-Cruz, and G. Lorenzo. 2011. “Particulate Matter Studies by the Manila Observatory." PCA General Assembly, Manila.

Slovic, P., B. Fischhoff, and S. Lichtenstein. 1985. “Characterizing Perceived Risk.” In Kates, R. W., C. Hohenhemser, and J. X. Kasperson (eds), Perilous Progress: Managing the Hazards of Technology. Westview: Boulder, CO, 92-125.

Tangphansankun, A., T. Okamura, and F. Nakamura. 2010. "Influences of Paratransit as a Feeder of Mass Transit System in Developing Countries based on Commuter Satisfaction." Journal of the Eastern Asia Society for Transportation Studies 8: 13411356.

Theodossiou, I. 1998. "The Effects of Low-Pay and Unemployment on Psychological Well-Being: A Logistic Regression Approach." Journal of Health Economics 17(1): 85-104.

Tittarelli, A., A. Borgini, M. Bertoldi, E. de Saeger, A. Ruprecht, R. Stefanoni, G. Tagliabue, P. Contiero, and P. Crosignani. 2008. "Estimation of Particle Mass Concentration in Ambient Air Using a Particle Counter." Atmospheric Environment 42: 8543-8548.

Villarin, J. T., J. Simpas, G. Lorenzo, and M. T. Cruz. 2014."Air Quality in Metro Manila, Philippines." In Kim Oanh, N. T. (ed.), Improving Air Quality in Asian Developing Countries: Compilation of Research Findings. NARENCA.

Wichmann, H. E., C. Spix, T. Tuch, G. Wolke, A. Peters, J. Heinrich, W. G. Kreyling, and J. Heyder. 2000. "Daily Mortality and Fine and Ultrafine Particles in Erfurt, Germany. Part 1: Role of Particle Number and Particle Mass." Health Effects Institute Research Report. 98: 5-86. 


\section{About the Authors}

Andra Charis Mijares (asmijares@katahira.com) received her Ph.D. in Civil Engineering from the Tokyo Institute of Technology in Japan in September 2015. She currently works as a project development consultant at Katahira \& Engineers International. Her interests include sustainable public transportation systems especially in developing countries and intelligent transportation systems.

MIo SUZUKI (mios@enveng.titech.ac.jp) is an Assistant Professor at the Department of Built Environment at the Tokyo Institute of Technology, from which she earned a Doctor of Engineering degree. She is interested in sustainable transportation and bicycle safety research.

TETSUo YAI (tyai@enveng.titech.ac.jp) is a Professor at the Department of Built Environment at the Tokyo Institute of Technology. He is the President of the Eastern Asia Society for Transportation Studies (EASTS). His major research fields are sustainable transportation and national, regional, and urban planning. 